This item was submitted to Loughborough's Institutional Repository (https://dspace.lboro.ac.uk/) by the author and is made available under the following Creative Commons Licence conditions.

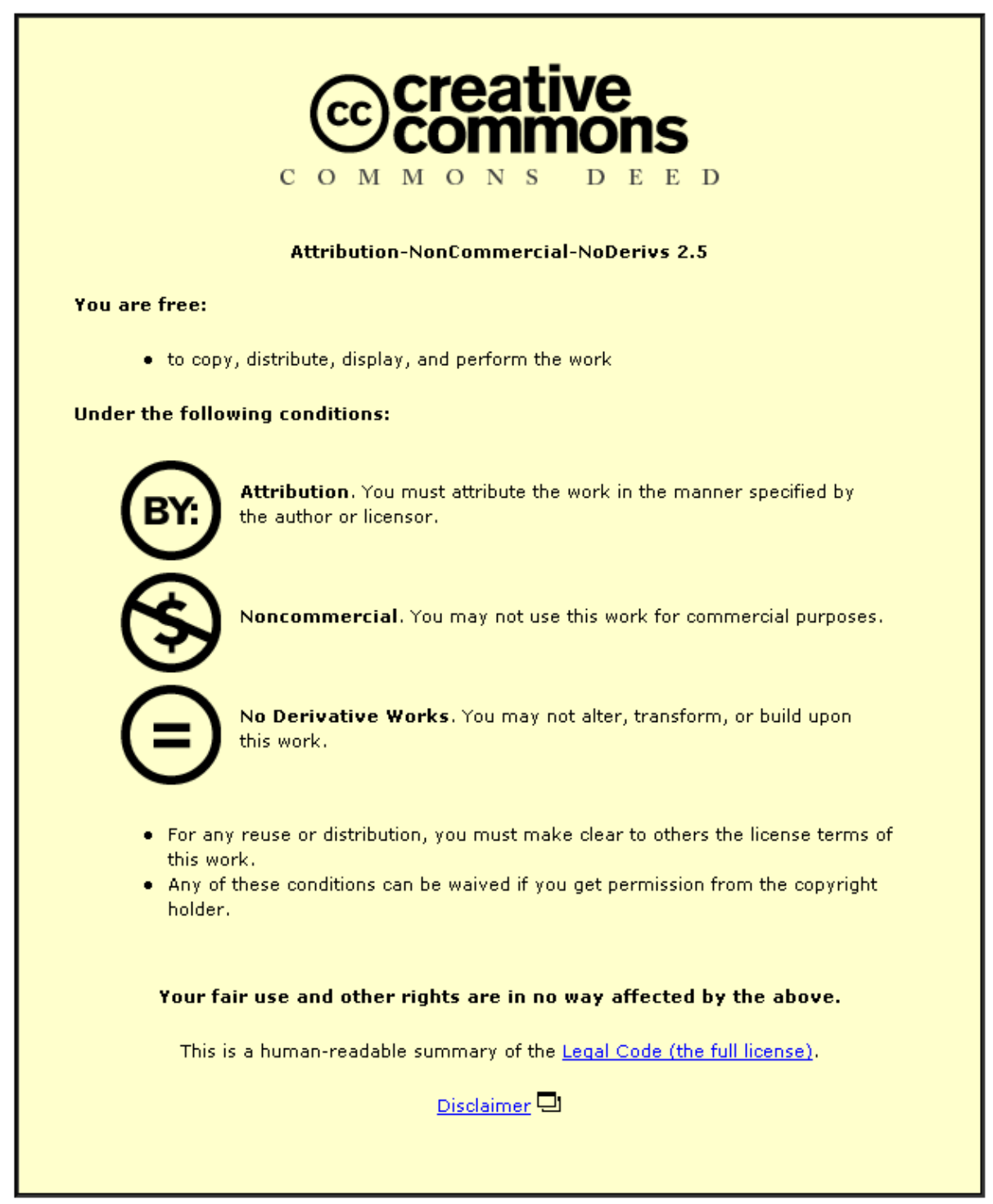

For the full text of this licence, please go to: http://creativecommons.org/licenses/by-nc-nd/2.5/ 


\title{
The relationship between bowling action classification and three-dimensional lower trunk motion in fast bowlers in cricket
}

\author{
Craig A. Ranson ${ }^{1}$, Angus F. Burnett ${ }^{2,3}$, Mark King ${ }^{4}$, Nitin Patel ${ }^{5}$, and Peter B. O’Sullivan ${ }^{2}$ \\ ${ }^{1}$ Physiotherapy, ECB National Cricket Performance Centre, Loughborough University, UK \\ ${ }^{2}$ School of Physiotherapy, Curtin University of Technology, Perth, WA, Australia \\ ${ }^{3}$ School of Exercise, Biomedical and Health Sciences, Edith Cowan University, Western Australia \\ ${ }^{4}$ School of Sport and Exercise Sciences, Loughborough University, UK \\ ${ }^{5}$ Pinner Road Physiotherapy, Harrow, UK
}

\begin{abstract}
Lower back injuries, specifically lumbar stress fractures, account for the most lost playing time in professional cricket. The aim of this study was to investigate the relationship between the current fast bowling action classification system and potentially injurious kinematics of the lower trunk during fast bowling. Three-dimensional kinematic data were collected from 50 male professional fast bowlers during a standing active range of motion trial and three fast bowling trials. Seventyfour percent of the fast bowlers used a mixed bowling action attributable to having shoulder counter-rotation greater than $30^{\circ}$. There was no difference in the proportion of available lower trunk extension, contralateral side-flexion and ipsilateral rotation range of motion used during fast bowling by mixed and non-mixed action bowlers. The greatest proportion of lower trunk extension $(26 \%)$, contralateral side-flexion $(131 \%)$ and ipsilateral rotation $(79 \%)$ was utilised during the front-foot contact phase of the fast bowling delivery stride. It is proposed that the combination of end range lower trunk positioning, especially side-flexion, and high ground reaction (compressive) forces during the front foot contact phase are the most important pathomechanical factors in the typical pattern of contralateral side lumbar stress injuries seen in this population.
\end{abstract}

Keywords: fast bowling, cricket, trunk kinematics, lower back injuries

\section{Introduction}

Lower back injuries to fast bowlers in cricket results in the greatest amount of lost playing time amongst professional cricketers (Orchard et al., 2002, Newman, 2003). Lumbar stress injuries (pars interarticularis stress reaction and stress fracture) are the most prevalent injury type and in fast bowlers these occur predominantly on the opposite side to the bowling arm (Elliott et al., 1992, Ranawat et al., 2003, Gregory et al., 2004, Ranson et al., 2005). In addition, a unique pattern of multi-level, non-dominant side, chronic pars interarticularis stress reactions are highly prevalent in this population, and when compared to age-matched athletic individuals, fast bowlers have a higher prevalence of multiple level lumbar disc degeneration (Ranson et al., 2005).

Several studies published since the late 1980's have identified and examined risk factors for lower back injury and lumbar radiological abnormalities commonly seen in fast bowlers (Elliott et al., 1986, Foster et al., 1989, Elliott et al., 1992, Elliott et al., 1993, Burnett et al., 1995, Burnett et al., 1996, Elliott and Khangure, 2002, Portus et al., 2004). It is believed that the development of lower back injury in fast bowlers is multifactorial, although, fast bowling technique has been the predominant area of investigation due the reported relationship between specific aspects of fast bowling technique and the appearance of radiological abnormalities (Elliott et al., 1992, Elliott et al., 1993, Burnett et al., 1996).

The fast bowling action begins during the delivery stride prior to ball release. The first critical event during the delivery stride is back foot contact (BFC) when the bowler's back foot impacts the ground. This is followed by front-foot contact (FFC) then ball release (BR). Fast bowling actions can be broadly categorised into one of four action types: front-on, side-on, mid-way and mixed and this is determined according to the alignment of the 
shoulders at BFC and the amount of shoulder counter-rotation (SCR) during the delivery stride (Elliott et al., 1992, Burnett et al., 1995, Clarke and Morris, 1996, Portus et al., 2004). SCR is defined as the change in the shoulder alignment angle from a relatively front-on alignment at BFC (Figure 1a) to the most side-on shoulder alignment (Figure 1b) during the delivery stride (minimum shoulder angle). Several classification systems have been described in the literature with SCR thresholds for the mixed action being as low as $10^{\circ}$ (Elliott et al., 1992, Glazier et al., 2000) and as high as $40^{\circ}$ (Foster et al., 1989). The fast bowling classification systems currently in use within the cricket associations in the United Kingdom and Australia state that any bowler that has greater than $30^{\circ} \mathrm{SCR}$ is classified as having a mixed action (Clarke and Morris, 1996, Portus et al., 2004). Further, when measuring shoulder and pelvic alignment, bowlers can be classified as having a mixed action when the pelvic to shoulder separation angle at BFC exceeds $30^{\circ}$ (Stockhill and Bartlett, 1992, Burnett et al., 1995, Portus et al., 2004).

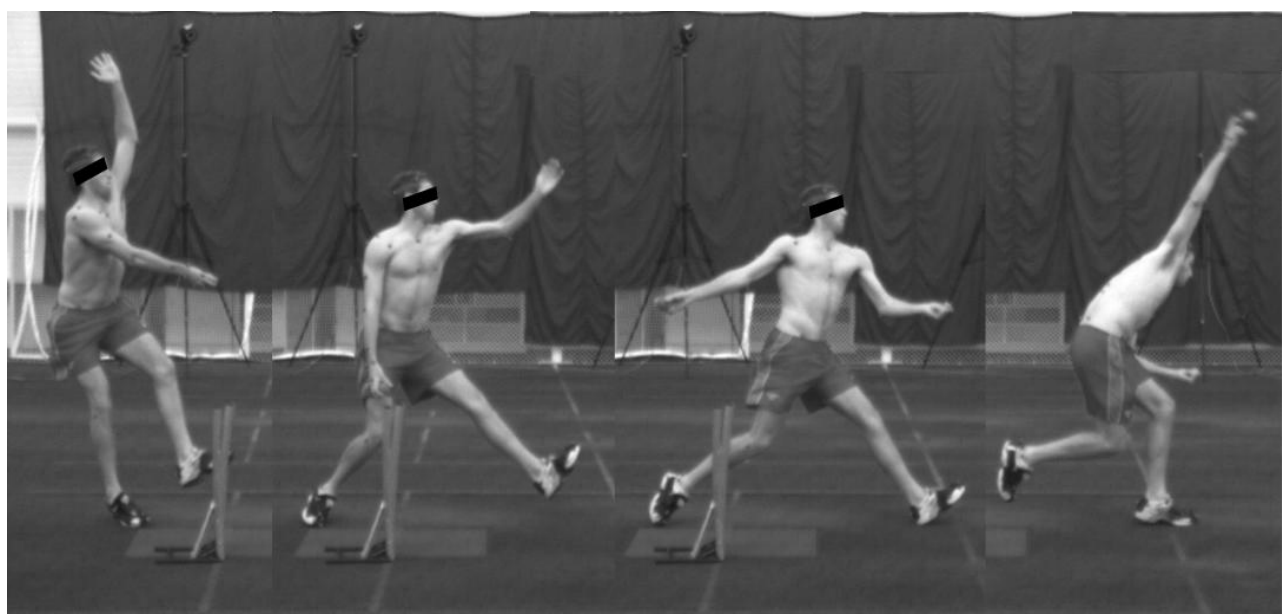

Figure 1. The delivery stride of fast bowling a) Back foot contact b) Minimum shoulder angle c) Front foot contact d) Ball release.

Several studies have reported an association between lower back injury and the mixed bowling action (Foster et al., 1989, Elliott et al., 1992, Elliott et al., 1993, Burnett et al., 1996). Specifically, Portus et al. (2004), in a retrospective study of elite Australian fast bowlers, reported that bowlers who previously suffered lower back soft tissue injuries had non-significantly larger pelvic to shoulder separation angles at BFC. Furthermore, SCR values were found to be significantly higher in bowlers who had suffered lumbar stress fractures when compared to non trunk-injured bowlers. However, the precise mechanism behind the relationship between high SCR and elevated rates of lumbar spine stress in mixed action bowlers is presently unknown. To date, only one study (Burnett et al., 1998), has investigated aspects of the 3D kinematics of the lower trunk spine during fast bowling. This study revealed that although variables used to classify fast bowling action type occurred between BFC and FFC, the movements most likely to place the greatest mechanical load on the lumbar spine occurred between FFC and BR. Coincidently, this is also the phase of the bowling action where peak ground reaction forces are produced (Elliott et al., 1986, Foster et al., 1989, Hurrion et al., 2000).

Chosa et al. (2004) found that unilateral pars interarticularis stress was greatest under combinations of compression with lumbar extension, compression with lumbar side-flexion to the same side, and compression with lumbar rotation to the opposite side. Further, it is known that the available ROM of lumbar axial rotation is reduced when the spine is in end range extension (Haberl et al., 2004, Burnett et al., 2006), therefore implying increased stiffening of 
the spine when it is positioned near the limits of its physiological ROM. Panjabi (1992) terms this zone of high stiffness towards end range the "elastic zone of motion". Repeated motion within this "elastic zone", combined with the large ground reaction forces during FFC, may well provide the pathomechanical forces responsible for the unique pattern of contralateral side lower lumbar stress injuries.

Therefore, the aim of this study was to investigate the relationship between the current fast bowling action classification system and potentially injurious kinematics of the trunk during fast bowling. Trunk kinematic variables included those used to classify bowling action type i.e. shoulder and pelvic alignment, along with the proportion of lower trunk extension, side-flexion and axial rotation utilised during the delivery stride of fast bowling.

\section{Methods}

\section{Subjects and experimental protocol}

This study recruited 50 professional male fast bowlers from English County Cricket clubs. This sample represented approximately $25 \%$ of the professional fast bowlers playing first class County Cricket. Subjects were considered as fast bowlers by the England Cricket Board fast bowling coaches. The mean $( \pm \mathrm{s})$ age, height and weight of the subjects was $23 \pm 4$ years, $1.86 \pm 0.05 \mathrm{~m}$ and $86 \pm 8 \mathrm{~kg}$ respectively. Subjects were deemed fit to bowl by their County Physiotherapist and had all bowled three times per week, on average, in either practice sessions or matches during the current season. Ethical approval for this study was obtained from the Human Research Ethics Committees of the University of Nottingham, UK and Curtin University of Technology, Western Australia.

\section{Data Collection}

A 12 camera Vicon Motion Analysis System (Oxford, UK) operating at $120 \mathrm{~Hz}$ was used to capture a lower trunk range of motion (ROM) trial and six fast bowling trials for each bowler. These trials were maximum velocity deliveries that pitched in an area designated as a good line and length by a qualified fast bowling coach. Testing was conducted in the indoor practice facility at the England and Wales Cricket Board National Cricket Centre at Loughborough University. This facility allowed the subjects to bowl with their normal length run-up on a standard size artificial cricket pitch (Figure 2). Cameras were positioned around the bowling crease to cover a $7 \mathrm{~m} \times 3 \mathrm{~m} \times 3 \mathrm{~m}$ volume which was wand calibrated prior to data collection.

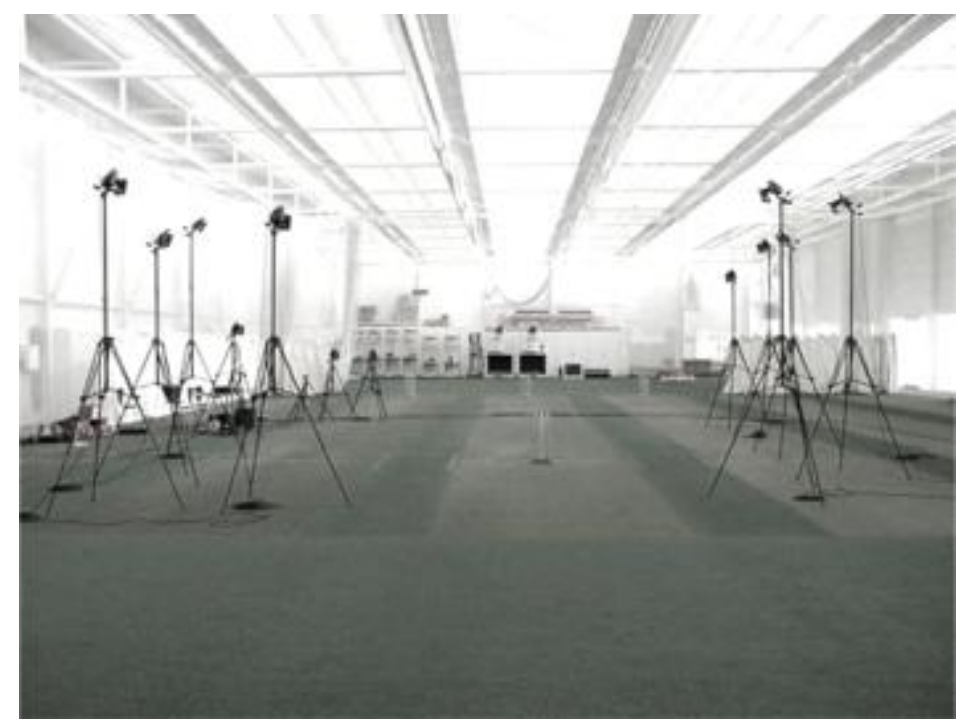

Figure 2. Experimental setup in the indoor cricket training facility. 
Thirty-one, $14 \mathrm{~mm}$ diameter, spherical reflective markers were attached to bony landmarks (standard Vicon Golem whole body marker set, OMG Plc, Oxford UK) using aerosol sports adhesive and double-sided tape. Seven of these markers were used to define two local reference frames in the pelvic and lower thorax regions of the trunk:Pelvic reference frame - markers were placed over the left and right anterior superior iliac spine (ASIS) and the left and right posterior superior iliac spine (PSIS).

Lower thorax reference frame - markers were placed over the xiphoid process at the distal end of the sternum and the spinous processes of T10 and L1.

The pelvic and lower thorax reference frames where used to quantify lower trunk kinematics during the ROM and fast bowling trials.

Markers were also attached to the right and left acromia, the head (four), arms (four on each arm) and legs (four on each leg) to allow whole body motion to be determined using Vicon BodyBuilder (OMG Plc, Oxford UK) software. A square of reflective tape $(2 \mathrm{~cm} \times$ $2 \mathrm{~cm}$ ) was also fixed to one side of the cricket ball to allow the instant of ball release and the ball velocity to be determined. Prior to testing subjects were given adequate time for their routine pre-bowling warm-up activities which included several warm-up deliveries.

For the standing ROM trial the bowlers were given a demonstration and instruction in how to move to their end range of active lower trunk flexion and extension, left and right side-flexion, and left and right axial rotation. The instructions were: "From an upright standing position, with your arms held out horizontally to the side, bend as far as you can forwards, then as far as you can backwards. Then, again starting from the upright position, bend over as far as you can to the left, then to the right. Finally, move back to upright and rotate as far as you can to the left, then to the right." Subjects then practised this motion so that the investigators were confident that they were moving to the end of their trunk ROM in each direction.

\section{Data processing}

Three-dimensional marker locations were reconstructed using the Vicon Workstation (OMG Plc, Oxford UK) software and all six bowling trials were manually labelled before selecting the best three (maximum velocity trials with minimal marker loss) of each bowler for further analysis along with the ROM trial. The instants of back foot contact (BFC) (Figure 1a) and front foot contact (FFC) (Figure 1c) were defined as the first image where the appropriate foot came in contact with the ground based upon visual inspection of the horizontal time histories of the heel and toe markers. Ball release (BR) was defined as the image closest to release of the ball (Figure 1d).

Determining the two local reference frames which defined lower trunk kinematics required an origin and two vectors to be defined for each coordinate system. Both reference frames were defined with the first axis equal to vector 1, the second axis equal to the crossproduct of vector 2 and vector 1 and the third axis equal to the cross-product of the first and second axes such that a right handed orthogonal reference frame was produced with the $\mathrm{X}$ axis (Figure 3). 


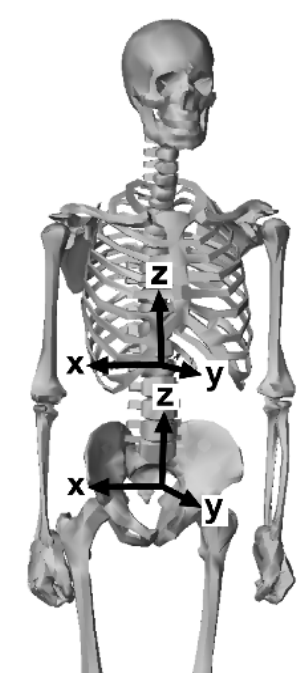

Figure 3. Local orthogonal reference frames for the pelvis and lower thorax used to determine lower trunk kinematics.

\section{Bowling Action Classification}

The bowling action of each subject was classified as one of four bowling types according to the shoulder angle and pelvic to shoulder separation angle (Table 1). Most of the previous studies that have classified bowling actions according to shoulder, or shoulder and pelvic alignment have viewed these segments in the horizontal plane (Stockhill and Bartlett, 1992, Burnett et al., 1995, Portus et al., 2004). Consequently, in the current study the shoulder angle was determined by projecting the $3 \mathrm{D}$ alignment of the left and right acromia onto a horizontal plane $\left(180^{\circ}=\right.$ side-on, $270^{\circ}=$ shoulders aligned with the bowling crease $)$. The pelvic angle was determined by projecting the $\mathrm{X}$-axis of the pelvic reference frame onto a horizontal plane $\left(180^{\circ}=\right.$ side-on, $270^{\circ}=$ pelvis aligned with the bowling crease $)$. The pelvic to shoulder separation angle was then defined as the shoulder angle minus the pelvic angle (Burnett et al., 1995, Burnett et al., 1998, Portus et al., 2004).

Table 1. Fast bowling action classification variables and their typical values used to define fast bowling action types (Portus et al., 2004)

\begin{tabular}{cccc}
\hline $\begin{array}{c}\text { Action } \\
\text { Type }\end{array}$ & $\begin{array}{c}\text { Shoulder Angle } \\
\text { at Back Foot } \\
\text { Contact }\end{array}$ & $\begin{array}{c}\text { Shoulder Counter- } \\
\text { Rotation }\end{array}$ & $\begin{array}{c}\text { Pelvic-Shoulder } \\
\text { Separation at } \\
\text { Back Foot Contact }\end{array}$ \\
\hline Front-on & $>240^{\circ}$ & $<30^{\circ}$ & $<30^{\circ}$ \\
Midway & $240^{\circ}-210^{\circ}$ & $<30^{\circ}$ & $<30^{\circ}$ \\
Side-on & $>210^{\circ}$ & $<30^{\circ}$ & $<30^{\circ}$ \\
Mixed & $\mathrm{n} / \mathrm{a}$ & $\geq 30^{\circ}$ & $\geq 30^{\circ}$ \\
\hline
\end{tabular}

\section{Lower Trunk Kinematics}

The orientation of the lower thorax reference frame relative to the pelvic reference frame was defined using Cardan angles to quantify flexion-extension about a lateral pelvic axis, side-flexion about a floating frontal axis, and axial rotation about the lower thorax longitudinal axis for both the ROM and bowling trials (Cole et al., 1993, Burnett et al., 1998). A 'neutral' upright anatomical position was identified for each bowler in the ROM trial and 
all subsequent measures were then expressed relative to this posture. As the absolute angular position about each orthopaedic axes for the lower trunk relative the pelvic reference frame in the standing neutral posture was not equal to zero matrix algebra procedures outlined by Burnett et al. (1998) were used to adjust the neutral posture to $(0,0,0)$. Maximal lower trunk motion of the variables thought most likely to contribute to contralateral side lumbar stress injuries i.e. extension, contralateral side-flexion and ipsilateral axial rotation, (Chosa et al., 2004) were determined for the ROM trial, and each bowling trial.

The time histories of each kinematic descriptor were fitted using quintic splines (Wood and Jennings, 1979). The closeness of fit at each point was based on the difference between the descriptor value and the average value from the two adjacent times (Yeadon and King, 2002).

\section{Reliability of the Kinematic Variables}

Intra-class correlation (ICC), and relative SEM (\%SEM) statistics (Norton et al., 2000) were calculated to determine the inter-trial variability of each of the variables used to determine the bowling action type, and each of the lower trunk kinematic variables obtained during the three fast bowling trials. To further quantify the inter-trial variability and the random noise in the data average standard deviation and standard error of the mean values were calculated for each variable for each subject.

All action classification and trunk kinematic variables had high ICC values (range 0.86 - 0.97) (Table 2). Low \%SEM values were found for all variables (range 1.5 - 9.1) apart from, pelvic to shoulder separation at BFC and lower trunk extension which had moderate $\%$ SEM values (18.8 \& 17.2 respectively) (Table 2 ). In addition, the overall average standard deviation for all action classification and trunk kinematic variables was low $\left(2.8^{\circ}\right.$, range $1.8^{\circ}$ $\left.5^{\circ}\right)$, as was the overall average standard error of the mean was $\left(1.6^{\circ}\right.$, range $\left.1.1^{\circ}-2.9^{\circ}\right)$. As a result of the high reliability of these variables, data from three trials for each dependent variable in the study were averaged to provide representative values for each bowler. The maximum lower trunk extension, contralateral side-flexion and ipsilateral rotation utilised during the fast bowling trails was then expressed as a percentage of the maximum ROM achieved during the ROM trial

Table 2. Reliability indices (ICC and \%SEM) for variables used to determine fast bowling action type and trunk kinematic variables of interest. All mean (s) data are in degrees.

\begin{tabular}{|c|c|c|c|c|}
\hline Kinematics & Variable & $\operatorname{Mean}^{\circ}(\mathrm{s})$ & ICC & $\% \mathrm{SEM}$ \\
\hline \multirow{4}{*}{$\begin{array}{c}\text { Action } \\
\text { Classification }\end{array}$} & Shoulder Angle at Back Foot Contact & $234(18)$ & 0.97 & 1.5 \\
\hline & Minimum Shoulder Angle & $194(10)$ & 0.9 & 1.5 \\
\hline & Shoulder Counter-Rotation & $41(16)$ & 0.97 & 6.7 \\
\hline & Back Foot Contact Pelvic-Shoulder Separation & $23(13)$ & 0.89 & 18.8 \\
\hline \multirow{3}{*}{ Lower Trunk } & Extension & $9(6)$ & 0.93 & 17.2 \\
\hline & Contralateral Side-Flexion & $34(7)$ & 0.88 & 6.7 \\
\hline & Ipsilateral Rotation & $32(8)$ & 0.86 & 9.1 \\
\hline Whole Trunk & Maximum Pelvic-Shoulder Separation & $45(9)$ & 0.93 & 5.3 \\
\hline
\end{tabular}


The instants of the delivery stride where bowlers obtained the minimum shoulder angle, maximum pelvic to shoulder separation angle, maximum lower trunk extension, maximum contralateral side-flexion and maximum ipsilateral rotation, relative to the time of FFC, were also determined.

\section{Statistical analysis}

Independent t-tests were used to determine if there was any difference in the proportion of lower trunk ROM utilised by mixed and non-mixed action (front-on, midway and side-on) bowlers. Non-mixed action bowlers were pooled as these action types have previously been considered 'safer' for the lower back than the mixed action (Foster et al., 1989, Burnett et al., 1996, Burnett et al., 1998, Elliott and Khangure, 2002). Effect sizes were also calculated for this comparison with effect sizes of less than 0.2 considered small, 0.2 to 0.5 medium and 0.5 to 0.8 large (Cohen, 1988). Further, Pearson's product moment correlation coefficients were used to determine whether any association was evident between SCR and pelvic to shoulder separation angles, in addition to selected lower trunk kinematic variables. Correlation coefficients between 0.2 to 0.4 were considered weak, 0.4 to 0.7 as moderate and greater than 0.7 as strong (Johnston, 2000). All analyses were conducted using SPSS V11.0 for Windows (SPSS Inc., Chicago, Illinois). The level of statistical significance was set at $p<0.05$.

\section{Results}

For the purposes of this study only shoulder angle at BFC and the magnitude of SCR were used to classify the type of bowling action utilised as the third variable used to classify action type, pelvic to shoulder separation at BFC, has been shown not to be associated with pars interarticularis stress injury in fast bowlers (Portus et al., 2004). Using the above criteria, 39 of the 50 bowlers $(78 \%)$ were determined to have used a mixed action, while nine eight $(16 \%)$ used a mid-way action and three $(6 \%)$ used a side-on action. No bowler was deemed to have used a front-on bowling action. It should be noted that if pelvic to shoulder separation at BFC was included as an action classification criteria there would have been little difference in the number of bowlers in each action type. Specifically, only a further two of the midway bowlers would have been classified as mixed as they had a pelvic to shoulder separation at $\mathrm{BFC}$ of over $30^{\circ}$.

The percentage of lower trunk extension, contralateral side-flexion and ipsilateral axial rotation used by bowlers of each action type is displayed in Table 3. There was no significant difference in the percentage of lower trunk extension, contralateral side-flexion and ipsilateral axial rotation used by the mixed action bowlers when compared to the nonmixed action bowlers (Table 3 ) although large effect sizes were found for contralateral lower trunk contralateral side-flexion $(d=0.66)$ and ipsilateral rotation $(d=0.57)$. Also, there was no difference $(\mathrm{t}=0.117, p=0.91)$ in the minimum shoulder angle obtained by the mixed (mean $194^{\circ}, s=9$ ) and non-mixed action (mean $\left.193^{\circ}, s=11\right)$ bowlers. 
Table 3. Mean (s) of available lower trunk movement expressed as a percentage of range of motion for extension, ipsilateral rotation and contralateral side-flexion utilised by bowlers of each action type, and grouped averages for non-mixed and all bowlers. All mean (s) data are in degrees.

\begin{tabular}{ccccccc}
\hline & \multicolumn{5}{c}{ Mean (s) percentage of lower trunk ROM } \\
\hline Action Type & Extension & $\begin{array}{c}\text { Contralateral } \\
\text { Side-Flexion }\end{array}$ & $\begin{array}{c}\text { Ipsilateral } \\
\text { Rotation }\end{array}$ \\
\hline Mixed & 27 & $(21)$ & 136 & $(36)$ & 81 & $(19)$ \\
Midway & 14 & $(17)$ & 118 & $(27)$ & 76 & $(23)$ \\
Side-on & 38 & $(16)$ & 108 & $(34)$ & 52 & $(13)$ \\
All Non-mixed & 20 & $(19)$ & 115 & $(28)$ & 69 & $(23)$ \\
All bowlers & 26 & $(21)$ & 131 & $(35)$ & 79 & $(20)$ \\
\hline $\begin{array}{c}\text { Mixed v Non Mixed }(t \\
\text { value })\end{array}$ & 0.978 & 1.757 & 1.792 \\
\hline $\begin{array}{c}\text { Mixed von Mixed }(P \\
\text { value })\end{array}$ & 0.33 & & 0.09 & 0.08 \\
\hline \begin{tabular}{c} 
Effect Size $(d$ value $)$ \\
\hline
\end{tabular} & 0.35 & & 0.66 & 0.57 \\
\hline
\end{tabular}

NB. Statistical comparison was only conducted between the groups shown in bold

A strong correlation $(r=0.86, p=0.00)$ was found between the shoulder angle at $\mathrm{BFC}$ and the magnitude of SCR. Further, there was a moderate correlation between the pelvic to shoulder separation angle at BFC and shoulder alignment at BFC $(r=0.52, p=0.00)$, along with a weak correlation between pelvic to shoulder separation angle at BFC and SCR $(r=$ $0.44, p=0.00)$. A significant, weak correlation was found between SCR and ipsilateral lower trunk rotation $(r=0.34, p=0.02)$. There was no correlation between SCR and the proportion of lower trunk extension or side flexion. Furthermore, no correlation was found between the pelvic to shoulder separation angle at BFC and the proportion of ROM of the lower trunk kinematic variables of interest (Table 4).

Table 4. Correlations, $r$ ( $P$ value), between Shoulder Counter-Rotation and the Pelvic to Shoulder Separation angle at Back Foot Contact (BFC), and the lower trunk kinematic variables of interest. Significant Correlations in bold.

\begin{tabular}{|c|c|c|c|c|c|}
\hline & $\begin{array}{c}\% \text { ROM } \\
\text { Extension }\end{array}$ & $\begin{array}{c}\% \mathrm{ROM} \\
\text { Contralateral } \\
\text { Side Flexion }\end{array}$ & $\begin{array}{l}\% \text { ROM } \\
\text { Ipsilateral } \\
\text { Rotation }\end{array}$ & $\begin{array}{c}\text { Shoulder } \\
\text { Angle at } \\
\text { Back Foot } \\
\text { Contact } \\
\end{array}$ & $\begin{array}{l}\text { Shoulder } \\
\text { Counter- } \\
\text { Rotation }\end{array}$ \\
\hline $\begin{array}{l}\text { Shoulder } \\
\text { Counter- } \\
\text { Rotation }\end{array}$ & $-0.04(0.78)$ & $0.18(0.21)$ & $0.34(0.02)$ & $0.86(0.00)$ & - \\
\hline $\begin{array}{c}\text { Pelvic to } \\
\text { Shoulder } \\
\text { Separation at } \\
\text { BFC }\end{array}$ & $-0.21(0.15)$ & $0.14(0.32)$ & $0.19(0.19)$ & $0.52(0.00)$ & $0.44(0.00)$ \\
\hline
\end{tabular}


The minimum shoulder angle typically occurred just prior to FFC whilst the maximum pelvic to shoulder separation occurred, on average, 0.03 seconds after FFC (Figure 4a). Maximum lower trunk extension took place, on average, 0.01 seconds after FFC whilst maximum ipsilateral rotation and contralateral side-flexion occurred slightly later in the delivery stride at an average of 0.04 and 0.05 seconds after FFC respectively (Figure 4b).
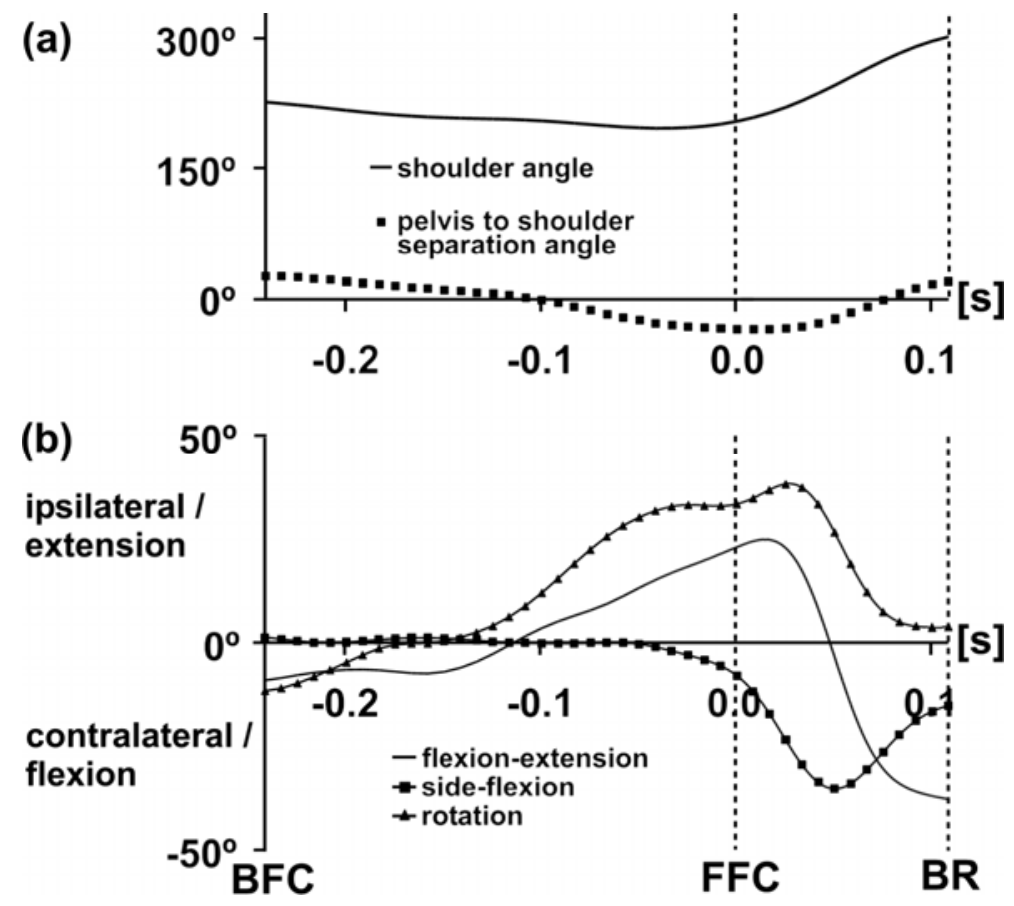

Figure 4. a) Typical shoulder angles and pelvic to shoulder separation angles, and b) lower trunk flexionextension, side-flexion and rotation angles during the delivery stride of fast bowling. Delivery stride events are back foot contact (BFC), front foot contact (FFC) and ball release (BR).

\section{Discussion}

Originally, fast bowling classification systems were designed to broadly describe bowling technique and biomechanical factors affecting performance (Elliott and Foster, 1984, Elliott et al., 1986). However, these systems have evolved in an attempt to identify fast bowlers at risk of low back injury (Foster et al., 1989, Burnett et al., 1996, Portus et al., 2004). Despite initiatives aimed at enabling coaches to recognize potentially 'unsafe' action types, the prevalence of low back injuries, particularly contralateral side lumbar stress injuries, remains high (Orchard et al., 2002, Newman, 2003). In addition, the mechanism by which supposedly dangerous action types result in excessive stress on the contralateral side lumbar pars interarticularis is unclear.

The aim of this study was to investigate the relationship between variables used in the fast bowling action classification system currently employed in the United Kingdom (Clarke and Morris, 1996) and Australia (Portus et al., 2004) and potentially injurious kinematics of the lower trunk during fast bowling. As reported in other studies that have used a similar classification system (Elliott and Khangure, 2002, Gray et al., 2003, Portus et al., 2004), a high proportion of the professional fast bowlers in this study were deemed to use what is currently considered to be an unsafe action type i.e. the mixed action. Further, $78 \%$ of bowlers were classified as having a mixed action (SCR $>30^{\circ}$ ) because they had high SCR, a variable previously associated with lumbar stress fracture (Foster et al., 1989, Elliott et al., 
1992, Portus et al., 2004). If the intent of action classification systems is to identify 'at risk' bowlers, the current system is unlikely to be able to sensitively identify those at increased risk of lumbar stress injury due to the large number of bowlers using a mixed technique.

In this study all bowlers tended to adopt a relatively side-on alignment of the shoulders just prior to FFC (Figure $1 \mathrm{~b}$ ) regardless of shoulder alignment (front-on, midway or side-on) at BFC (Figure 1a). The implication of this is that under the current action classification system the front-on classification appears redundant as all 16 bowlers in this study who had a front-on shoulder alignment at BFC had SCR greater than $30^{\circ}$ and were therefore classified as 'mixed'. Based on the above findings, a review of the current methods of identifying potentially dangerous bowling technique should be considered.

Pars interarticularis stress is reported to be greatest under combinations of compression with lumbar extension, compression with lumbar side-flexion to the same side, and compression with lumbar rotation to the opposite side (Chosa et al., 2004). The current action classification variables; shoulder angle at $\mathrm{BFC}$, pelvic to shoulder separation at $\mathrm{BFC}$ and SCR, are measured during the BFC to FFC phase. In this phase the lower trunk is typically positioned in a relatively neutral posture when compared to just prior to FFC and through to ball release (Figures $1 \& 3 \mathrm{~b}$ ). Contact forces on the contralateral facet joints of the lumbar spine are likely to be low between BFC and FFC as the front foot is not in contact with the ground. Temporal analysis revealed that maximal lower trunk extension, side-flexion and rotation typically occur just after FFC (Figure 4b). Therefore, the combination of these trunk kinematics and high ground reaction forces that occur just after FFC (Elliott et al., 1986, Foster et al., 1989, Hurrion et al., 2000, Portus et al., 2004) are likely to result in maximal stresses on the contralateral side lumbar pars interarticulari during this phase of the bowling action. In accordance with the findings of Burnett et al. (1998), bowlers in this study with greater than $30^{\circ} \mathrm{SCR}$ did not use a significantly greater proportion of lower trunk extension, ipsilateral rotation and contralateral side-flexion when compared to non-mixed action bowlers. Therefore, there does not appear to be a pathomechanical basis for large SCR resulting in increased risk of stress injury to the contralateral side lumbar spine pars interarticularis.

Hyperextension of the lumbar spine is thought to be the mechanism of injury in other sports with a high rate of lumbar stress fractures such as gymnastics (Sward, 1992) and American Football (Ferguson et al., 1974). Previous authors have suggested that excessive SCR may force the lumbar spine into hyperextension (Annear et al., 1989, Elliott et al., 1992, Bartlett, 2003). However, fast bowlers in this study utilised only a relatively small proportion of their available lower trunk extension ROM (26\%) which questions the importance of this movement in the aetiology of lumbar stress injuries within this population.

The greatest proportion of lower trunk ROM utilised by the fast bowlers in this study was in contralateral side-flexion. During the FFC phase of the delivery stride fast bowlers utilised approximately 1.3 times the amount of side-flexion they obtained during the standing ROM trial. This was probably due to the inertia of upper body and trunk causing significantly greater "elastic zone" motion of the spine whilst bowling as opposed to slow active sideflexion in standing. However, such a large amount of contralateral lower trunk side-flexion was not expected as maximum side-flexion occurred during a phase of the bowling action where the lower trunk was also extended and rotated to the ipsilateral side. These coupled movements should have reduced the range of available side-flexion (Burnett et al., 2006). Therefore, it might be concluded that this position of extreme contralateral lower trunk sideflexion, in combination with large ground reaction forces, is the most significant stressor of the contralateral side lumbar pars interarticulari (Panjabi, 1992, Chosa et al., 2004).

SCR during the delivery stride of fast bowling has previously been proposed to be an indicator of spinal torsional stress (Foster et al., 1989, Elliott et al., 1993, Elliott et al., 
2002) and in this study a significant correlation, albeit weak, was found between SCR and the proportion of ipsilateral lower trunk rotation. However, using SCR to directly estimate the degree of torsional stress in the lumbar spine may be problematic. This is due to the fact that SCR is a significantly removed derivative of whole trunk rotation (Stockill and Bartlett, 1996) which occurs during the BFC to FFC phase of the bowling action whilst maximal lower trunk rotation occurs much later. Specifically maximal ipsilateral trunk rotation occurs, during the FFC to BR phase when the shoulders are rotating towards the contralateral side (Figure 4). Furthermore, a mechanical modelling study has indicated that rotational stresses alone are unlikely to be the major pathomechanical factor in lumbar stress injury (Chosa et al., 2004). Additionally, in comparison to contralateral side-flexion, the proportion of ipsilateral lower trunk rotation utilised by fast bowlers was relatively low (79\% versus $131 \%$ ). This again suggests a greater importance of side-flexion than rotation or extension in the production of contralateral side lumbar pars interarticularis stress.

Maximum contralateral side-flexion, coupled with extension and ipsilateral rotation, coincides with large ground reaction (compressive) forces that occur soon after FFC (Elliott et al., 1986, Foster et al., 1989, Hurrion et al., 2000, Portus et al., 2004). This combination of lower trunk movements is the likely mechanical aetiology of the high prevalence of stress injuries that occur in the contralateral low lumbar spine of fast bowlers (Elliott et al., 1992, Gregory et al., 2004, Ranson et al., 2005). The likely pathomechanics of lumbar stress injuries in fast bowlers does not appear to be primarily related to excessive SCR, the action characteristic previously reported to be related to low back injury (Elliott et al., 1992, Burnett et al., 1996, Elliott and Khangure, 1999, Portus et al., 2004). Prevention of low back injuries in fast bowlers in cricket may require coaches to pay greater attention to spinal positioning during the FFC phase of the delivery stride, especially the magnitude of contralateral sideflexion.

\section{Conclusion}

A very high percentage of fast bowlers in this, and other studies, have been classified as having a mixed bowling action whilst no bowlers in this study were classified as front-on. Further, fast bowling action characteristics currently used to identify potentially dangerous action types do not appear to be directly related to the likely pathomechanics of contralateral side lumbar stress injuries. It is proposed that extreme lower trunk kinematics, especially contralateral side-flexion, during the early part of the FFC phase of the bowling action is likely to be the most important mechanical factor in the aetiology of this type of injury. However, prospective studies are required to determine the relationship between lower trunk kinematics and lumbar spine stress injuries in fast bowlers.

\section{References}

Annear, P., Chakera, T., Foster, D. and Hardcastle, P. (1989). Pars interarticularis stress and disc degeneration in cricket's potent strike force: The fast bowler. Australian and New Zealand Journal of Surgery, 62, 768-773.

Bartlett, R. M. (2003). The science and medicine of cricket: an overview and update. Journal of Sports Sciences, 21, 733-752.

Burnett, A., Elliott, B. C. and Marshall, R. (1995). The effect of a 12 over spell on fast bowling technique in cricket. Journal of Sports Sciences, 13, 329-341.

Burnett, A., Barrett, C., Marshall, R., Elliott, B. C. and Day, R. (1998). Three-dimensional measurement of lumbar spine kinematics for fast bowlers in cricket. Clinical Biomechanics, 13, 574-583. 
Burnett, A., Khangure, M., Elliott, B. C., Foster, D., Marshall, R. and Hardcastle, P. (1996). Thoracolumbar disc degeneration in young fast bowlers in cricket: A follow up study. Clinical Biomechanics, 11, 305-310.

Burnett, A., O’Sullivan, P., Ankaberg, L., Gooding, M., Nelis, R., Offermann, F. and Persson, J. (2006). Lower lumbar spine axial rotation is reduced in end range sagittal postures when compared to a neutral spine posture. Submitted.

Chosa, E., Totoribe, K. and Tajima, N. (2004). A biomechanical study of lumbar spondylolysis based on a three-dimensional finite element method. Journal of Orthopaedic Research, 22, 158-163.

Clarke, D. and Morris, H. (Eds.) (1996). Cricket Coach's Manual, London: England \& Wales Cricket Board.

Cohen, J. (1988). Statistical power analysis for the behavioural sciences Hillsdale, NJ: Lawrence Earlbaum Associates.

Cole, G. K., Nigg, B. M., Ronsky, J. L. and Yeadon, M. R. (1993). Application of the joint coordinate system to three-dimensional joint attitude and movement representation: A standardization proposal. Journal of Biomechanical Engineering, 115, 344-349.

Elliott, B., Wallis, R., Sakurai, S., Lloyd, D. and Besier, T. (2002). The measurement of shoulder alignment in cricket fast bowling. Journal of Sports Sciences, 20, 507-510.

Elliott, B. C. and Foster, D. (1984). A biomechanical analysis of the front-on and side-on fast bowling techniques. Journal of Human Movement Studies, 10, 83-94.

Elliott, B. C. and Khangure, M. (1999). The link between fast bowling technique and disc degeneration in young cricketers: Preliminary data from a follow-up study. In XVIIth International Society of Biomechanics Congress (edited by W. Herzog \& A. Jinha). Calgary, Canada: International Society of Biomechanics.

(2002). Disk degeneration and fast bowling in cricket: An intervention study. Medicine and Science in Sports and Exercise, 34, 1714-1718.

Elliott, B. C., Foster, D. and Gray, S. (1986). Biomechanics and physical factors affecting fast bowling. Australian Journal of Science and Medicine in Sport, 18, 16-21.

Elliott, B. C., Hardcastle, P., Burnett, A. and Foster, D. (1992). The influence of fast bowling and physical factors on radiological features in high performance fast bowlers. Sports Medicine Training and Rehabilitation, 3, 113-130.

Elliott, B. C., Davis, J., Khangure, M., Hardcastle, P. and Foster, D. (1993). Disc degeneration and the young fast bowler in cricket. Clinical Biomechanics, 8, 227234.

Ferguson, R. J., McMaster, J. H. and Stanitski, C. L. (1974). Low back pain in college football linemen. Journal of Sports Medicine, 2, 63-69.

Foster, D., John, D., Elliott, B. C., Ackland, T. and Fitch, K. (1989). Back injuries to fast bowlers in cricket: A prospective study. British Journal of Sports Medicine, 23, 150154.

Glazier, P. S., Paradisis, G. P. and Cooper, S. (2000). Anthropometric and kinematic influences on release speed in men's fast-medium bowling. Journal of Sports Sciences, 18, 1013-1021.

Gray, J., Derman, W., Vaughan, C. L. and Noakes, T. D. (2003). The Classification System for Cricket Fast Bowling Actions Revised. In Science and Medicine in Cricket: A collection of papers from the Second World Congress of Science and Medicine in Cricket (edited by R. A. Stretch, T. D. Noakes \& C. L. Vaughan). Cape Town, South Africa: comPress. 
Gregory, P., Batt, M. E. and Kerslake, R. W. (2004). Comparing spondylolysis in cricketers and soccer players. British Journal of Sports Medicine, 38, 737-742.

Haberl, H., Cripton, P. A., Orr, T. E., Beutler, T., Frei, H. and Lanksch, W. R. (2004). Kinematic response of lumbar functional spinal units to axial torsion with and without superimposed compression and flexion/extension. European Spine Journal, 13, 560-566.

Hurrion, P. D., Dyson, R. and Hale, T. (2000). Simultaneous measurement of back and front foot ground reaction forces during the same delivery stride of the fast-medium bowler. Journal of Sports Sciences, 18, 993-997.

Johnston, I. (2000). An Introductory Handbook on Probability, Statistics, and Excel. http://www.mala.bc.ca/ johnstoi/maybe/maybe4.htm

Newman, D. (2003). A prospective survey of injuries at first class counties in England and Wales 2001 and 2002 seasons. In Science and Medicine in Cricket: A collection of papers from the Second World Congress of Science and Medicine in Cricket (edited by R. A. Stretch, T. D. Noakes \& C. L. Vaughan). Cape Town, South Africa: comPress.

Norton, K., Marfell-Jones, M., Whittingham, N., Kerr, D., Carter, L., Saddington, K. and Gore, C. (2000). Anthropometric Assessment Protocols. In Physiological Tests for Elite Athletes (edited by C. Gore), pp.66-85 Lower Mitcham: South Australia: Human Kinetics.

Orchard, J., James, T., Alcott, E., Carter, S. and Farhart, P. (2002). Injuries in Australian cricket at first class level 1995/1996 to 2000/2001. British Journal of Sports Medicine, 36, 270-274.

Panjabi, M. (1992). The stabilizing system of the spine. Part 1. Function, dysfunction, application and enhancement. Journal of Spinal Disorders, 5, 383-389.

Portus, M. R., Mason, B. R., Elliot, B. C., Pfitzner, M. C. and Done, R. P. (2004). Technique factors related to ball release speed and trunk injuries in high performance cricket fast bowlers. Sports Biomechanics, 3, 263-283.

Ranawat, V. S., Dowell, J. K. and Heywood-Waddington, M. B. (2003). Stress fractures of the lumbar pars interarticularis in athletes: a review based on long-term results in 18 professional cricketers. Injury, 34, 915-919.

Ranson, C., Kerslake, R., Burnett, A., Batt, M. and Abdi, S. (2005). Magnetic resonance imaging of the lumbar spine of asymptomatic professional fast bowlers in cricket. Journal of bone and Joint Surgery - Br, 87-B, 1111-1116.

Stockhill, N. and Bartlett, R. (1992). A three-dimensional cinematographical analysis of the techniques of international and English County Cricket fast bowlers. In Xth Symposium of the International Society of Biomechanics in Sports (edited by R. Rodano, G. Ferrigno \& G. C. Santambrogio). Milan: Edi Ermes.

Stockill, N. and Bartlett, R. (1996). Possible errors in measurement of shoulder alignment using 3-D cinematography. In XIVth International Symposium on Biomechanics in Sport. (edited by J. Abrantes). Portugal: Edicoes.

Sward, L. (1992). The thoracolumbar spine in young elite athletes. Current concepts on the effects of physical training. Sports Medicine, 13, 357-364.

Wood, G. A. and Jennings, L. S. (1979). On the use of spline functions for data smoothing. Journal of Biomechanics, 12, 477-479.

Yeadon, M. R. and King, M. A. (2002). Evaluation of a torque driven simulation model of tumbling. Journal of Applied Biomechanics, 18, 195-206. 
